\title{
THE CAUCHY PROBLEM FOR ONE-DIMENSIONAL FLOW OF A COMPRESSIBLE VISCOUS FLUID: STABILIZATION OF THE SOLUTION
}

\author{
Nermina Mujaković and Ivan DražIĆ \\ University of Rijeka, Croatia
}

\begin{abstract}
We analyze the Cauchy problem for non-stationary 1-D flow of a compressible viscous and heat-conducting fluid, assuming that it is in the thermodynamical sense perfect and polytropic. This problem has a unique generalized solution on $\mathbb{R} \times] 0, T[$ for each $T>0$. Supposing that the initial functions are small perturbations of the constants and using some a priori estimates for the solution independent of $T$, we prove a stabilization of the solution.
\end{abstract}

\section{INTRODUCTION}

In this paper we analyze the Cauchy problem for non-stationary 1-D flow of a compressible viscous and heat-conducting fluid. It is assumed that the fluid is thermodynamically perfect and polytropic. The same model has been mentioned in [1], where a global-in-time existence theorem for generalized solution is given without a rigorous proof. Here, we approach to our problem as the special case of the Cauchy problem for a micropolar fluid that is considered in [5] and [6]. Therefore we know that this problem has a unique generalized solution on $\mathbb{R} \times] 0, T$, for each $T>0$ and that the mass density and temperature are strictly positive.

Assuming that the initial functions are small perturbations of the constants, we first derive a priori estimates for a solution independent of $T$ and then analyze the behavior of the solution as $T \rightarrow \infty$. We use some ideas of Ya. I. Kanel' ([4]) applied to the case of stabilization of Hölder continuous solution for the same model.

2010 Mathematics Subject Classification. 46E35, 35B40, 35B45, 76N10.

Key words and phrases. Compressible viscous fluid, the Cauchy problem, stabilization. 


\section{Statement of the Problem And the main Result}

Let $\rho, v$ and $\theta$ denote, respectively, the mass density, velocity, and temperature of the fluid in the Lagrangean description. Supposing that in [5] the microrotation is equal to zero, we obtain the problem considered as follows:

$$
\begin{gathered}
\frac{\partial \rho}{\partial t}+\rho^{2} \frac{\partial v}{\partial x}=0 \\
\frac{\partial v}{\partial t}=\frac{\partial}{\partial x}\left(\rho \frac{\partial v}{\partial x}\right)-K \frac{\partial}{\partial x}(\rho \theta) \\
\frac{\partial \theta}{\partial t}=-K \rho \theta \frac{\partial v}{\partial x}+\rho\left(\frac{\partial v}{\partial x}\right)^{2}+D \frac{\partial}{\partial x}\left(\rho \frac{\partial \theta}{\partial x}\right)
\end{gathered}
$$

in $\mathbb{R} \times \mathbb{R}^{+}$, where $K$ and $D$ are positive constants. The equations (2.1)-(2.3) are, respectively, local forms of the conservation laws for the mass, momentum and energy ${ }^{1}$. We take the following non-homogeneous initial conditions:

$$
\begin{gathered}
\rho(x, 0)=\rho_{0}(x), \\
v(x, 0)=v_{0}(x), \\
\theta(x, 0)=\theta_{0}(x)
\end{gathered}
$$

for $x \in \mathbb{R}$, where $\rho_{0}, v_{0}$ and $\theta_{0}$ are given functions. We assume that there exist the constants $m, M \in \mathbb{R}^{+}$, such that

$$
m \leq \rho_{0}(x) \leq M, \quad m \leq \theta_{0}(x) \leq M, \quad x \in \mathbb{R} .
$$

In the papers [5] and [6] it was proved that for

$$
\rho_{0}-1, v_{0}, \theta_{0}-1 \in \mathrm{H}^{1}(\mathbb{R})
$$

the problem (2.1)-(2.6) has, for each $T \in \mathbb{R}^{+}$, a unique generalized solution

$$
(x, t) \mapsto(\rho, v, \theta)(x, t), \quad(x, t) \in \Pi=\mathbb{R} \times] 0, T[,
$$

with the properties:

$$
\begin{gathered}
\rho-1 \in \mathrm{L}^{\infty}\left(0, T ; \mathrm{H}^{1}(\mathbb{R})\right) \cap \mathrm{H}^{1}(\Pi), \\
v, \theta-1 \in \mathrm{L}^{\infty}\left(0, T ; \mathrm{H}^{1}(\mathbb{R})\right) \cap \mathrm{H}^{1}(\Pi) \cap \mathrm{L}^{2}\left(0, T ; \mathrm{H}^{2}(\mathbb{R})\right) .
\end{gathered}
$$

Using the results from [5] and [1] we can easily conclude that

$$
\theta, \rho>0 \text { in } \Pi \text {. }
$$

${ }^{1}$ Derivation of the equations (2.1)-(2.3) from the Eulerian description is given in [1], pp. 31-42. 
We denote by $\mathrm{B}^{k}(\mathbb{R}), k \in \mathbb{N}_{0}$, the Banach space

$$
\mathrm{B}^{k}(\mathbb{R})=\left\{u \in \mathrm{C}^{k}(\mathbb{R}): \lim _{|x| \rightarrow \infty}\left|D^{n} u(x)\right|=0,0 \leq n \leq k\right\}
$$

where $D^{n}$ is $n$-th derivative. The norm of the space $\mathrm{B}^{k}(\mathbb{R})$ is defined by

$$
\|u\|_{\mathrm{B}^{k}(\mathbb{R})}=\sup _{n \leq k}\left\{\sup _{x \in \mathbb{R}}\left|D^{n} u(x)\right|\right\} .
$$

From Sobolev's embedding theorem ([2, Chapter IV $]$ ) and the theory of vector-valued distributions ([3, pp. 467-480]) one can conclude that from (2.10) and (2.11) follows

$$
\rho-1 \in \mathrm{L}^{\infty}\left(0, T ; \mathrm{B}^{0}(\mathbb{R})\right) \cap \mathrm{C}\left([0, T] ; \mathrm{L}^{2}(\mathbb{R})\right),
$$

(2.16) $v, \theta-1 \in \mathrm{L}^{2}\left(0, T ; \mathrm{B}^{1}(\mathbb{R})\right) \cap \mathrm{C}\left([0, T] ; \mathrm{H}^{1}(\mathbb{R})\right) \cap \mathrm{L}^{\infty}\left(0, T ; \mathrm{B}^{0}(\mathbb{R})\right)$ and hence

$$
v, \theta-1 \in \mathrm{C}\left([0, T] ; \mathrm{B}^{0}(\mathbb{R})\right), \quad \rho \in \mathrm{L}^{\infty}(\Pi) .
$$

From (2.7) and (2.8) it is easy to see that there exist the constants $E_{1}$, $E_{2}, E_{3}, M_{1} \in \mathbb{R}^{+}, M_{1}>1$, such that

(2.18) $\frac{1}{2} \int_{\mathbb{R}} v_{0}^{2} d x+K \int_{\mathbb{R}}\left(\frac{1}{\rho_{0}}-\ln \frac{1}{\rho_{0}}-1\right) d x+\int_{\mathbb{R}}\left(\theta_{0}-\ln \theta_{0}-1\right) d x=E_{1}$,

$$
\begin{gathered}
\frac{1}{2} \int_{\mathbb{R}}\left(\left(v_{0}^{\prime}\right)^{2}+\left(\theta_{0}^{\prime}\right)^{2}\right) d x=E_{2}, \\
\frac{1}{2} \int_{\mathbb{R}} \frac{\left(\rho_{0}^{\prime}\right)^{2}}{\rho_{0}^{2}} d x+\int_{\mathbb{R}} v_{0}^{\prime} \ln \frac{1}{\rho_{0}} d x \leq E_{3}, \\
\sup _{|x|<\infty} \theta_{0}(x)<M_{1} .
\end{gathered}
$$

Suppose that the quantities $\underline{\eta}$ and $\bar{\eta}$ are such that $\underline{\eta}<0<\bar{\eta}$ and

$$
\int_{\underline{\eta}}^{0} \sqrt{e^{\eta}-1-\eta} d \eta=\int_{0}^{\bar{\eta}} \sqrt{e^{\eta}-1-\eta} d \eta=E_{5}
$$

where

$$
E_{5}=2 \sqrt{\frac{E_{1} E_{4}}{K}}, \quad E_{4}=2 \mu E_{1}\left(1+M_{1}+\frac{E_{3}}{E_{1}}\right), \quad \mu=\max \left\{\frac{K}{2 D}, 1\right\} .
$$

Let

$$
\underline{u}=\exp \underline{\eta}, \quad \bar{u}=\exp \bar{\eta} .
$$

The aim of this work is to prove the following theorem. 
THEOREm 2.1. Suppose that the initial functions satisfy (2.7), (2.8) and the following conditions:

$$
E_{1} \int_{\mathbb{R}}\left(v_{0}^{\prime}\right)^{2} d x<\left(\frac{D}{16} \frac{\underline{\bar{u}}}{\bar{u}}\right)^{2},
$$

$$
\begin{aligned}
& 2 E_{1}\left(E_{1} \frac{\bar{u}}{\underline{u}}\left(1+M_{1}\right) M_{1}\left(\left(16 E_{4}\right)^{2} \frac{\bar{u}}{\underline{u}}+\frac{K^{2}}{D} M_{1}\right)+2 K M_{1} E_{4}+E_{2}\right) \\
& <\min \left\{\left(\frac{D}{16} \frac{\underline{u}}{\bar{u}}\right)^{2},\left(\int_{1}^{M_{1}} \sqrt{s-1-\ln s} d s\right)^{2},\left(\int_{0}^{1} \sqrt{s-1-\ln s} d s\right)^{2}\right\}
\end{aligned}
$$

then

$$
\rho(x, t) \rightarrow 1, \quad v(x, t) \rightarrow 0, \quad \theta(x, t) \rightarrow 1, \text { when } t \rightarrow \infty,
$$

uniformly with respect to all $x \in \mathbb{R}$.

Remark 2.2. Conditions (2.25) and (2.26) mean that $E_{1}, E_{2}$ and $E_{3}$ are sufficiently small. In other words the initial functions are small perturbations of the constants.

In the proof of Theorem 2.1. we apply some ideas of [4], where a stabilization of the solution that is Hölder continuous was proved for the same model.

\section{A PRORI estimates For $\rho, v$ AND $\theta$}

Considering stabilization problem, one has to prove some a priori estimates for the solution independent of $T$, which is the main difficulty. Some of our considerations are similar to those of [4]. First we derive the energy equation for the solution of problem (2.1)-(2.3) under the conditions indicated above and we estimate the function $\rho^{-1}$.

Lemma 3.1. For each $t>0$ we have

$$
\begin{aligned}
\frac{1}{2} \int_{\mathbb{R}} v^{2} d x+\int_{\mathbb{R}}(\theta-\ln \theta-1) d x+K \int_{\mathbb{R}}\left(\frac{1}{\rho}-\ln \frac{1}{\rho}-1\right) d x \\
+\int_{0}^{t} \int_{\mathbb{R}}\left(\frac{\rho}{\theta}\left(\frac{\partial v}{\partial x}\right)^{2}+D \frac{\rho}{\theta^{2}}\left(\frac{\partial \theta}{\partial x}\right)^{2}\right) d x d \tau=E_{1},
\end{aligned}
$$

where $E_{1}$ is defined by (2.18).

Proof. Multiplying (2.1), (2.2) and (2.3), respectively, by $K \rho^{-1}(1-$ $\left.\rho^{-1}\right), v$ and $1-\theta^{-1}$, integrating by parts over $\mathbb{R}$ and over $] 0, t[$ and taking into account (2.15) and (2.16), after addition of the obtained equations we easily get equality (3.1) independently of $t$. 
Lemma 3.2. For each $t>0$ exist the strictly positive quantities $\underline{u}_{1}=$ $\underline{u}_{1}(\bar{\theta}(t))$ and $\bar{u}_{1}=\bar{u}_{1}(\bar{\theta}(t))$ such that

$$
\left.\underline{u}_{1} \leq \rho^{-1}(x, \tau) \leq \bar{u}_{1}, \quad(x, \tau) \in \mathbb{R} \times\right] 0, t[,
$$

where

$$
\bar{\theta}(t)=\sup _{(x, \tau) \in \mathbb{R} \times] 0, t[} \theta(x, \tau)
$$

Proof. We multiply (2.2) by $\frac{\partial}{\partial x} \ln \left(\frac{1}{\rho}\right)$, integrate over $\mathbb{R}$ and $] 0, t[$ and use the following equalities, which follow using (2.1) and (2.15)-(2.17):

$$
\frac{\partial}{\partial x}\left(\rho \frac{\partial v}{\partial x}\right) \frac{\partial}{\partial x}\left(\ln \frac{1}{\rho}\right)=\frac{1}{2} \frac{\partial}{\partial t}\left(\frac{\partial}{\partial x}\left(\ln \frac{1}{\rho}\right)\right)^{2}
$$

$$
\begin{aligned}
\int_{0}^{t} \int_{\mathbb{R}} \frac{\partial v}{\partial t} \frac{\partial}{\partial x}\left(\ln \frac{1}{\rho}\right) d x d \tau & =-\int_{0}^{t} \int_{\mathbb{R}} \frac{\partial^{2} v}{\partial x \partial t} \ln \frac{1}{\rho} d x d \tau \\
& =-\left.\int_{\mathbb{R}} \frac{\partial v}{\partial x} \ln \frac{1}{\rho} d x\right|_{0} ^{t}+\int_{0}^{t} \int_{\mathbb{R}} \rho\left(\frac{\partial v}{\partial x}\right)^{2} d x d \tau, \\
-\left.\int_{\mathbb{R}} \frac{\partial v}{\partial x} \ln \frac{1}{\rho} d x\right|_{0} ^{t} & =-\int_{\mathbb{R}} \frac{\partial}{\partial x} v \ln \frac{1}{\rho} d x+\int_{\mathbb{R}} v_{0}^{\prime} \ln \frac{1}{\rho_{0}} d x \\
& =\int_{\mathbb{R}} v \frac{\partial}{\partial x}\left(\ln \frac{1}{\rho}\right) d x+\int_{\mathbb{R}} v_{0}^{\prime} \ln \frac{1}{\rho_{0}} d x, \\
\frac{\partial}{\partial x}(\rho \theta) & =\frac{\partial \theta}{\partial x} \rho-\theta \rho^{2} \frac{\partial}{\partial x}\left(\frac{1}{\rho}\right) .
\end{aligned}
$$

We also take into account the following inequalities obtained by the Young's inequality

$$
\begin{aligned}
& \rho^{2} \frac{\partial \theta}{\partial x} \frac{\partial}{\partial x}\left(\frac{1}{\rho}\right) \leq \frac{1}{2} \frac{\rho}{\theta}\left(\frac{\partial \theta}{\partial x}\right)^{2}+\frac{1}{2} \theta \rho^{3}\left(\frac{\partial}{\partial x}\left(\frac{1}{\rho}\right)\right)^{2}, \\
& v \frac{\partial}{\partial x} \ln \left(\frac{1}{\rho}\right)=v \rho \frac{\partial}{\partial x}\left(\frac{1}{\rho}\right) \leq \frac{1}{4} \rho^{2}\left(\frac{\partial}{\partial x}\left(\frac{1}{\rho}\right)\right)^{2}+v^{2} .
\end{aligned}
$$

After simple transformations we get

$$
\begin{aligned}
& \frac{1}{4} \int_{\mathbb{R}} \rho^{2}\left(\frac{\partial}{\partial x}\left(\frac{1}{\rho}\right)\right)^{2} d x+\frac{K}{2} \int_{0}^{t} \int_{\mathbb{R}} \theta \rho^{3}\left(\frac{\partial}{\partial x}\left(\frac{1}{\rho}\right)\right)^{2} d x d \tau \\
& \leq \int_{\mathbb{R}} v^{2} d x+\frac{K}{2} \int_{0}^{t} \int_{\mathbb{R}} \frac{\rho}{\theta}\left(\frac{\partial \theta}{\partial x}\right)^{2} d x d \tau+\int_{0}^{t} \int_{\mathbb{R}} \rho\left(\frac{\partial v}{\partial x}\right)^{2} d x d \tau \\
& \quad+\int_{\mathbb{R}} v_{0}^{\prime} \ln \left(\frac{1}{\rho_{0}}\right) d x+\frac{1}{2} \int_{\mathbb{R}} \frac{1}{\rho_{0}^{2}}\left(\rho_{0}^{\prime}\right)^{2} d x
\end{aligned}
$$


for each $t>0$. Using (3.1) and (2.20) from (3.10) we obtain

$$
\frac{1}{4} \int_{\mathbb{R}} \rho^{2}\left(\frac{\partial}{\partial x}\left(\frac{1}{\rho}\right)\right)^{2} d x+\frac{K}{2} \int_{0}^{t} \int_{\mathbb{R}} \theta \rho^{3}\left(\frac{\partial}{\partial x}\left(\frac{1}{\rho}\right)\right)^{2} d x d \tau \leq K_{1}(\bar{\theta}(t)),
$$

where

$$
K_{1}(\bar{\theta}(t))=2 \mu E_{1}\left(1+\bar{\theta}(t)+\frac{E_{3}}{E_{1}}\right)
$$

and $\mu, E_{1}$ and $E_{3}$ are defined by (2.23), (2.18) and (2.20).

Now we define the increasing function $\psi$ by

$$
\psi(\eta)=\int_{0}^{\eta} \sqrt{e^{\xi}-1-\xi} d \xi
$$

One can conclude the following

$$
\left|\psi\left(\ln \frac{1}{\rho}\right)\right|=\left|\int_{0}^{\ln \frac{1}{\rho}} \psi^{\prime}(\xi) d \xi\right| \leq\left|\int_{\mathbb{R}} \psi^{\prime}\left(\ln \frac{1}{\rho}\right) \rho \frac{\partial}{\partial x}\left(\frac{1}{\rho}\right) d x\right| .
$$

Using (3.1), (3.11) and the Hölder's inequality we get

$$
\begin{aligned}
\left|\psi\left(\ln \frac{1}{\rho}\right)\right| & \leq\left(\int_{\mathbb{R}}\left(\frac{1}{\rho}-1-\ln \frac{1}{\rho}\right) d x\right)^{\frac{1}{2}}\left(\int_{\mathbb{R}} \rho^{2}\left(\frac{\partial}{\partial x}\left(\frac{1}{\rho}\right)\right)^{2} d x\right)^{\frac{1}{2}} \\
& \leq K_{2}(\bar{\theta}(t))
\end{aligned}
$$

where

$$
K_{2}(\bar{\theta}(t))=2 E_{1}\left(\frac{2 \mu}{K}\left(1+\bar{\theta}(t)+\frac{E_{3}}{E_{1}}\right)\right)^{\frac{1}{2}} .
$$

We can also easily conclude that there exist the quantities $\underline{\eta}_{1}=\underline{\eta}_{1}(\bar{\theta}(t))<0$ and $\bar{\eta}_{1}=\bar{\eta}_{1}(\bar{\theta}(t))>0$, such that

$$
\int_{\underline{\eta}_{1}}^{0} \sqrt{e^{\eta}-1-\eta} d \eta=\int_{0}^{\bar{\eta}_{1}} \sqrt{e^{\eta}-1-\eta} d \eta=K_{2}(\bar{\theta}(t)),
$$

where $K_{2}(\bar{\theta}(t))$ is defined by (3.16). Comparing (3.15) and (3.17) we conclude that

$$
\underline{u}_{1}=\exp \underline{\eta}_{1} \leq \rho^{-1}(x, \tau) \leq \exp \bar{\eta}_{1}=\bar{u}_{1}
$$

for $(x, \tau) \in \mathbb{R} \times] 0, t[$.

Now we find some estimates for the derivatives of the functions $v$ and $\theta$. 
LEMma 3.3. For each $t>0$ we have

$$
\begin{aligned}
& \frac{1}{2} \int_{\mathbb{R}}\left(\left(\frac{\partial v}{\partial x}\right)^{2}+\left(\frac{\partial \theta}{\partial x}\right)^{2}\right) d x \\
& +\frac{8 \bar{u}_{1}}{\left(D \underline{u}_{1}\right)^{2}} \int_{0}^{t}\left(\int_{\mathbb{R}}\left(\frac{\partial v}{\partial x}\right)^{2} d x\right)^{2}\left(K_{4}(\bar{\theta}(\tau))-\int_{\mathbb{R}}\left(\frac{\partial v}{\partial x}\right)^{2} d x\right) d \tau \\
& +\frac{D}{8} \int_{0}^{t} \int_{\mathbb{R}} \rho\left(\frac{\partial^{2} \theta}{\partial x^{2}}\right)^{2} d x d \tau \leq K_{3}(\bar{\theta}(t))
\end{aligned}
$$

where

$$
\begin{gathered}
K_{4}(\bar{\theta}(t))=\left(\frac{D \underline{u}_{1}}{16 \bar{u}_{1} \sqrt{E_{1}}}\right)^{2}, \\
K_{3}(\bar{\theta}(t))=E_{1} \frac{\bar{u}_{1}}{\underline{u}_{1}}(1+\bar{\theta}(t)) \bar{\theta}(t)\left(\left(16 K_{1}(\bar{\theta}(t))\right)^{2} \frac{\bar{u}_{1}}{\underline{u}_{1}}+\frac{K^{2}}{D} \bar{\theta}(t)\right) \\
+2 K \bar{\theta}(t) K_{1}(\bar{\theta}(t))+E_{2} .
\end{gathered}
$$

Proof. Multiplying equations (2.2) and (2.3), respectively, by $-\frac{\partial^{2} v}{\partial x^{2}}$ and $-\frac{\partial^{2} \theta}{\partial x^{2}}$, integrating over $\left.\mathbb{R} \times\right] 0, t[$ and using the following equality

$$
-\int_{0}^{t} \int_{\mathbb{R}} \frac{\partial v}{\partial t} \frac{\partial^{2} v}{\partial x^{2}} d x d \tau=\left.\frac{1}{2} \int_{\mathbb{R}}\left(\frac{\partial v}{\partial x}\right)^{2} d x\right|_{0} ^{t}
$$

that is satisfied for the function $\theta$ as well, after addition of the obtained equalities we find that

$$
\begin{aligned}
& \left.\frac{1}{2} \int_{\mathbb{R}}\left(\left(\frac{\partial v}{\partial x}\right)^{2}+\left(\frac{\partial \theta}{\partial x}\right)^{2}\right) d x\right|_{0} ^{t}+\int_{0}^{t} \int_{\mathbb{R}} \rho\left(\frac{\partial^{2} v}{\partial x^{2}}\right)^{2} d x d \tau \\
& \quad+D \int_{0}^{t} \int_{\mathbb{R}} \rho\left(\frac{\partial^{2} \theta}{\partial x^{2}}\right)^{2} d x d \tau \\
& =-\int_{0}^{t} \int_{\mathbb{R}} \frac{\partial \rho}{\partial x} \frac{\partial v}{\partial x} \frac{\partial^{2} v}{\partial x^{2}} d x d \tau+K \int_{0}^{t} \int_{\mathbb{R}} \rho \frac{\partial \theta}{\partial x} \frac{\partial^{2} v}{\partial x^{2}} d x d \tau \\
& \quad+K \int_{0}^{t} \int_{\mathbb{R}} \theta \frac{\partial \rho}{\partial x} \frac{\partial^{2} v}{\partial x^{2}} d x d \tau+K \int_{0}^{t} \int_{\mathbb{R}} \rho \theta \frac{\partial v}{\partial x} \frac{\partial^{2} \theta}{\partial x^{2}} d x d \tau \\
& \quad-\int_{0}^{t} \int_{\mathbb{R}} \rho\left(\frac{\partial v}{\partial x}\right)^{2} \frac{\partial^{2} \theta}{\partial x^{2}} d x d \tau-D \int_{0}^{t} \int_{\mathbb{R}} \frac{\partial \rho}{\partial x} \frac{\partial \theta}{\partial x} \frac{\partial^{2} \theta}{\partial x^{2}} d x d \tau
\end{aligned}
$$


222

N. MUJAKOVIĆ AND I. DRAŽIĆ

Using (3.18), (3.11) and the following inequality

$$
\left(\frac{\partial v}{\partial x}\right)^{2} \leq 2\left(\int_{\mathbb{R}}\left(\frac{\partial v}{\partial x}\right)^{2}\right)^{\frac{1}{2}}\left(\int_{\mathbb{R}}\left(\frac{\partial^{2} v}{\partial x^{2}}\right)^{2}\right)^{\frac{1}{2}}
$$

that holds for the function $\frac{\partial \theta}{\partial x}$ as well, and applying the Young's inequality with a sufficiently small parameter on the right-hand side of (3.23) we come to the estimates as follows:

$(3.25)$

$$
\begin{aligned}
& \left|\int_{0}^{t} \int_{\mathbb{R}} \frac{\partial \rho}{\partial x} \frac{\partial v}{\partial x} \frac{\partial^{2} v}{\partial x^{2}} d x d \tau\right| \\
& \leq \int_{0}^{t} \int_{\mathbb{R}} \frac{1}{\rho}\left(\frac{\partial \rho}{\partial x}\right)^{2}\left(\frac{\partial v}{\partial x}\right)^{2} d x d \tau+\frac{1}{4} \int_{0}^{t} \int_{\mathbb{R}} \rho\left(\frac{\partial^{2} v}{\partial x^{2}}\right)^{2} d x d \tau \\
& \leq \frac{2 \bar{u}_{1}^{\frac{1}{2}}}{\underline{u}_{1}} \int_{0}^{t}\left(\int_{\mathbb{R}} \frac{1}{\rho^{2}}\left(\frac{\partial \rho}{\partial x}\right)^{2} d x\right)\left(\int_{\mathbb{R}}\left(\frac{\partial v}{\partial x}\right)^{2} d x\right)^{\frac{1}{2}}\left(\int_{\mathbb{R}} \rho\left(\frac{\partial^{2} v}{\partial x^{2}}\right)^{2} d x\right)^{\frac{1}{2}} d \tau \\
& +\frac{1}{4} \int_{0}^{t} \int_{\mathbb{R}} \rho\left(\frac{\partial^{2} v}{\partial x^{2}}\right)^{2} d x d \tau \\
& \leq \frac{5}{16} \int_{0}^{t} \int_{\mathbb{R}} \rho\left(\frac{\partial^{2} v}{\partial x^{2}}\right)^{2} d x d \tau+\left(16 K_{1}(\bar{\theta}(t))\right)^{2} \frac{\bar{u}_{1}}{\underline{u}_{1}^{2}} \int_{0}^{t} \int_{\mathbb{R}}\left(\frac{\partial v}{\partial x}\right)^{2} d x d \tau \\
& \leq \frac{5}{16} \int_{0}^{t} \int_{\mathbb{R}} \rho\left(\frac{\partial^{2} v}{\partial x^{2}}\right)^{2} d x d \tau \\
& +\left(16 K_{1}(\bar{\theta}(t)) \frac{\bar{u}_{1}}{\underline{u}_{1}}\right)^{2} \bar{\theta}(t) \int_{0}^{t} \int_{\mathbb{R}} \frac{\rho}{\theta}\left(\frac{\partial v}{\partial x}\right)^{2} d x d \tau, \\
& \left|K \int_{0}^{t} \int_{\mathbb{R}} \rho \frac{\partial \theta}{\partial x} \frac{\partial^{2} v}{\partial x^{2}} d x d \tau\right| \\
& \leq K^{2} \int_{0}^{t} \int_{\mathbb{R}} \rho\left(\frac{\partial \theta}{\partial x}\right)^{2} d x d \tau+\frac{1}{4} \int_{0}^{t} \int_{\mathbb{R}} \rho\left(\frac{\partial^{2} v}{\partial x^{2}}\right)^{2} d x d \tau \\
& \leq \frac{K^{2} \bar{u}_{1} \bar{\theta}^{2}(t)}{\underline{u}_{1}} \int_{0}^{t} \int_{\mathbb{R}} \frac{\rho}{\theta^{2}}\left(\frac{\partial \theta}{\partial x}\right)^{2} d x d \tau+\frac{1}{4} \int_{0}^{t} \int_{\mathbb{R}} \rho\left(\frac{\partial^{2} v}{\partial x^{2}}\right)^{2} d x d \tau, \\
& \left|K \int_{0}^{t} \int_{\mathbb{R}} \theta \frac{\partial \rho}{\partial x} \frac{\partial^{2} v}{\partial x^{2}} d x d \tau\right| \\
& (3.27) \leq K^{2} \int_{0}^{t} \int_{\mathbb{R}} \frac{\theta^{2}}{\rho}\left(\frac{\partial \rho}{\partial x}\right)^{2} d x d \tau+\frac{1}{4} \int_{0}^{t} \int_{\mathbb{R}} \rho\left(\frac{\partial^{2} v}{\partial x^{2}}\right)^{2} d x d \tau \\
& \leq K^{2} \bar{\theta}(t) \int_{0}^{t} \int_{\mathbb{R}} \theta \rho^{3}\left(\frac{\partial}{\partial x}\left(\frac{1}{\rho}\right)\right)^{2} d x d \tau+\frac{1}{4} \int_{0}^{t} \int_{\mathbb{R}} \rho\left(\frac{\partial^{2} v}{\partial x^{2}}\right)^{2} d x d \tau,
\end{aligned}
$$




$$
\begin{aligned}
\mid K & \int_{0}^{t} \int_{\mathbb{R}} \rho \theta \frac{\partial v}{\partial x} \frac{\partial^{2} \theta}{\partial x^{2}} d x d \tau \mid \\
(3.28) \leq & \frac{K^{2}}{D} \int_{0}^{t} \int_{\mathbb{R}} \theta^{2} \rho\left(\frac{\partial v}{\partial x}\right)^{2} d x d \tau+\frac{D}{4} \int_{0}^{t} \int_{\mathbb{R}} \rho\left(\frac{\partial^{2} \theta}{\partial x^{2}}\right)^{2} d x d \tau \\
\leq & \frac{K^{2} \bar{u}_{1} \bar{\theta}^{3}(t)}{D \underline{u}_{1}} \int_{0}^{t} \int_{\mathbb{R}} \frac{\rho}{\theta}\left(\frac{\partial v}{\partial x}\right)^{2} d x d \tau+\frac{D}{4} \int_{0}^{t} \int_{\mathbb{R}} \rho\left(\frac{\partial^{2} \theta}{\partial x^{2}}\right)^{2} d x d \tau, \\
& \left|\int_{0}^{t} \int_{\mathbb{R}} \rho\left(\frac{\partial v}{\partial x}\right)^{2} \frac{\partial^{2} \theta}{\partial x^{2}} d x d \tau\right| \\
\leq & \frac{1}{D} \int_{0}^{t} \int_{\mathbb{R}} \rho\left(\frac{\partial v}{\partial x}\right)^{4} d x d \tau+\frac{D}{4} \int_{0}^{t} \int_{\mathbb{R}} \rho\left(\frac{\partial^{2} \theta}{\partial x^{2}}\right)^{2} d x d \tau \\
\leq & \frac{2 \bar{u}_{1}^{\frac{1}{2}}}{D \underline{u}_{1}} \int_{0}^{t}\left(\int_{\mathbb{R}}\left(\frac{\partial v}{\partial x}\right)^{2} d x\right)^{\frac{3}{2}}\left(\int_{\mathbb{R}} \rho\left(\frac{\partial^{2} v}{\partial x^{2}}\right)^{2} d x\right)^{\frac{1}{2}} d \tau \\
& +\frac{D}{4} \int_{0}^{t} \int_{\mathbb{R}} \rho\left(\frac{\partial^{2} \theta}{\partial x^{2}}\right)^{2} d x d \tau \\
\leq & \frac{8 \bar{u}_{1}}{D^{2} \underline{u}_{1}^{2}} \int_{0}^{t}\left(\int_{\mathbb{R}}\left(\frac{\partial v}{\partial x}\right)^{2} d x\right)^{3} d \tau+\frac{1}{8} \int_{0}^{t} \int_{\mathbb{R}} \rho\left(\frac{\partial^{2} v}{\partial x^{2}}\right)^{2} d x d \tau \\
& +\frac{D}{4} \int_{0}^{t} \int_{\mathbb{R}} \rho\left(\frac{\partial^{2} \theta}{\partial x^{2}}\right)^{2} d x d \tau,
\end{aligned}
$$

(3.30)

$$
\begin{aligned}
\left|D \int_{0}^{t} \int_{\mathbb{R}} \frac{\partial \rho}{\partial x} \frac{\partial \theta}{\partial x} \frac{\partial^{2} \theta}{\partial x^{2}} d x d \tau\right| & \leq D \int_{0}^{t} \int_{\mathbb{R}} \frac{1}{\rho}\left(\frac{\partial \rho}{\partial x}\right)^{2}\left(\frac{\partial \theta}{\partial x}\right)^{2} d x d \tau+\frac{D}{4} \int_{0}^{t} \int_{\mathbb{R}} \rho\left(\frac{\partial^{2} \theta}{\partial x^{2}}\right)^{2} d x d \tau \\
\leq & \frac{2 D \bar{u}_{1}^{\frac{1}{2}}}{\underline{u}_{1}} \int_{0}^{t}\left(\int_{\mathbb{R}} \rho\left(\frac{\partial^{2} \theta}{\partial x^{2}}\right)^{2} d x\right)^{\frac{1}{2}}\left(\int_{\mathbb{R}}\left(\frac{\partial \theta}{\partial x}\right)^{2} d x\right)^{\frac{1}{2}} \int_{\mathbb{R}} \frac{1}{\rho^{2}}\left(\frac{\partial \rho}{\partial x}\right)^{2} d x d \tau \\
& +\frac{D}{4} \int_{0}^{t} \int_{\mathbb{R}} \rho\left(\frac{\partial^{2} \theta}{\partial x^{2}}\right)^{2} d x d \tau \\
\leq & \frac{128 D K_{1}^{2}(\bar{\theta}(t)) \bar{\theta}^{2}(t) \bar{u}_{1}^{2}}{\underline{u}_{1}^{2}} \int_{0}^{t} \frac{\rho}{\theta^{2}}\left(\frac{\partial \theta}{\partial x}\right)^{2} d x d \tau \\
& +\frac{3 D}{8} \int_{0}^{t} \int_{\mathbb{R}} \rho\left(\frac{\partial^{2} \theta}{\partial x^{2}}\right)^{2} d x d \tau .
\end{aligned}
$$



follows

Taking into account (3.25)-(3.30), (3.1), (3.11) and (2.19) from (3.23)

$$
\begin{aligned}
\frac{1}{2} \int_{\mathbb{R}} & {\left[\left(\frac{\partial v}{\partial x}\right)^{2}+\left(\frac{\partial \theta}{\partial x}\right)^{2}\right] d x+\frac{1}{16} \int_{0}^{t} \int_{\mathbb{R}} \rho\left(\frac{\partial^{2} v}{\partial x^{2}}\right)^{2} d x d \tau } \\
& +\frac{D}{8} \int_{0}^{t} \int_{\mathbb{R}} \rho\left(\frac{\partial^{2} \theta}{\partial x^{2}}\right)^{2} d x d \tau \\
\leq & \frac{8 \bar{u}_{1}}{D^{2} \underline{u}_{1}^{2}} \int_{0}^{t}\left(\int_{\mathbb{R}}\left(\frac{\partial v}{\partial x}\right)^{2} d x\right)^{3} d \tau+K_{3}(\bar{\theta}(t)),
\end{aligned}
$$

where $K_{3}(\bar{\theta}(t))$ is defined by (3.21). We also get another important inequality by estimating the integral $\int_{\mathbb{R}} \rho\left(\frac{\partial^{2} v}{\partial x^{2}}\right)^{2} d x$. We have

$$
\begin{aligned}
\int_{\mathbb{R}}\left(\frac{\partial v}{\partial x}\right)^{2} d x & =-\int_{\mathbb{R}} v \frac{\partial^{2} v}{\partial x^{2}} d x \\
& \leq \bar{u}_{1}^{\frac{1}{2}}\left(\int_{\mathbb{R}} v^{2} d x\right)^{\frac{1}{2}}\left(\int_{\mathbb{R}} \rho\left(\frac{\partial^{2} v}{\partial x^{2}}\right)^{2} d x\right)^{\frac{1}{2}} \\
& \leq\left(2 E_{1} \bar{u}_{1}\right)^{\frac{1}{2}}\left(\int_{\mathbb{R}} \rho\left(\frac{\partial^{2} v}{\partial x^{2}}\right)^{2} d x\right)^{\frac{1}{2}}
\end{aligned}
$$

Consequently,

$$
\int_{\mathbb{R}} \rho\left(\frac{\partial^{2} v}{\partial x^{2}}\right)^{2} d x \geq\left(2 E_{1} \bar{u}_{1}\right)^{-1}\left(\int_{\mathbb{R}}\left(\frac{\partial v}{\partial x}\right)^{2} d x\right)^{2} .
$$

Inserting (3.33) into (3.31) we obtain

$$
\begin{aligned}
& \frac{1}{2} \int_{\mathbb{R}}\left[\left(\frac{\partial v}{\partial x}\right)^{2}+\left(\frac{\partial \theta}{\partial x}\right)^{2}\right] d x \\
& +\frac{8 \bar{u}_{1}}{D^{2} \underline{u}_{1}^{2}} \int_{0}^{t}\left(\int_{\mathbb{R}}\left(\frac{\partial v}{\partial x}\right)^{2} d x\right)^{2}\left[\left(\frac{D \underline{u}_{1}}{16 \bar{u}_{1} E_{1}^{\frac{1}{2}}}\right)^{2}-\int_{\mathbb{R}}\left(\frac{\partial v}{\partial x}\right)^{2} d x\right] d \tau \\
& +\frac{D}{8} \int_{0}^{t} \int_{\mathbb{R}} \rho\left(\frac{\partial^{2} \theta}{\partial x^{2}}\right)^{2} d x d \tau \leq K_{3}(\bar{\theta}(t))
\end{aligned}
$$

and (3.18) is satisfied.

Similarly as in [4], in the continuation we use the above results, as well as the conditions of Theorem 2.1. We derive the estimates for the solution $(\rho, v, \theta)$ of problem (2.1)-(2.8) defined by (2.9)-(2.12) in the domain $\Pi=$ $\mathbb{R} \times] 0, T[$, for arbitrary $T>0$. 
Taking into account assumption (2.21) and the fact that $\theta \in C(\bar{\Pi})$ (see (2.17)) we have the following alternatives: either

$$
\sup _{(x, t) \in \Pi} \theta(x, t)=\bar{\theta}(T) \leq M_{1},
$$

or there exists $t_{1}, 0<t_{1}<T$, such that

$$
\bar{\theta}(t)<M_{1} \text { for } 0 \leq t<t_{1}, \quad \bar{\theta}\left(t_{1}\right)=M_{1} .
$$

Now we assume that (3.36) is satisfied and we will show later, that because of the choice of the constants $E_{1}, E_{2}, E_{3}$ and $M_{1}$ (the conditions of Theorem 2.1), (3.36) is impossible.

Because $K_{2}(\bar{\theta}(t))$, defined by (3.16), increases with increasing $\bar{\theta}(t)$ we can easily conclude that

$$
K_{2}(\bar{\theta}(t))<K_{2}\left(M_{1}\right) \text { for } 0 \leq t<t_{1}
$$

and $K_{2}\left(M_{1}\right)=E_{5}$. Therefore we have

$$
\underline{u}<\underline{u}_{1}(\bar{\theta}(t)), \quad \bar{u}>\bar{u}_{1}(\bar{\theta}(t))
$$

where $\underline{u}, \bar{u}$ and $\underline{u}_{1}(\bar{\theta}(t)), \bar{u}_{1}(\bar{\theta}(t))$ are defined by $(2.22)-(2.23)$ and (3.17)(3.18), respectively. The quantity $K_{4}(\bar{\theta}(t))$, defined by (3.20), decreases with increasing $\bar{\theta}(t)$ and for $\bar{\theta}\left(t_{1}\right)=M_{1}$ it becomes

$$
K_{4}\left(M_{1}\right)=\left(\frac{D \underline{u}}{16 \bar{u} E_{1}^{\frac{1}{2}}}\right)^{2} .
$$

Taking into account the assumption (2.25) of Theorem 2.1 and the following inclusion (See (2.16))

$$
\frac{\partial v}{\partial x} \in \mathrm{C}\left([0, T] ; \mathrm{L}^{2}(\mathbb{R})\right)
$$

we have again two alternatives: either

$$
\int_{\mathbb{R}}\left(\frac{\partial v}{\partial x}\right)^{2}(x, t) d x \leq K_{4}\left(M_{1}\right) \text { for } t \in\left[0, t_{1}\right],
$$

or there exists $t_{2}, \quad 0<t_{2}<t_{1}$, such that

$$
\int_{\mathbb{R}}\left(\frac{\partial v}{\partial x}\right)^{2}(x, t) d x<K_{4}\left(M_{1}\right) \text { for } 0 \leq t<t_{2},
$$

and

$$
\int_{\mathbb{R}}\left(\frac{\partial v}{\partial x}\right)^{2}\left(x, t_{2}\right) d x=K_{4}\left(M_{1}\right) \text { for } t_{2}<t_{1} .
$$

Now, we assume that (3.42)-(3.43) are satisfied. Then we have

$$
\bar{\theta}(t)<M_{1}, \quad K_{4}\left(M_{1}\right)<K_{4}(\bar{\theta}(t)) \text { for } t \in\left[0, t_{2}\right] .
$$


Taking into account (3.44), from (3.19), for $t=t_{2}$, we obtain

$$
\int_{\mathbb{R}}\left(\frac{\partial v}{\partial x}\right)^{2}(x, t) d x \leq 2 K_{3}\left(\bar{\theta}\left(t_{2}\right)\right), \quad 0 \leq t \leq t_{2} .
$$
holds

Since $K_{3}(\bar{\theta}(t))$, defined by (3.21), increases with the increase of $\bar{\theta}(t)$, it

$$
K_{3}(\bar{\theta}(t)) \leq K_{3}\left(M_{1}\right), \quad t \in\left[0, t_{2}\right]
$$

Using condition $(2.26)$ we get

$$
2 K_{3}(\bar{\theta}(t))<K_{4}\left(M_{1}\right), \quad t \in\left[0, t_{2}\right],
$$

and conclude that

$$
\int_{\mathbb{R}}\left(\frac{\partial v}{\partial x}\right)^{2}\left(x, t_{2}\right) d x<K_{4}\left(M_{1}\right) .
$$

This inequality contradicts (3.43). Consequently, the only case possible is when

$$
t_{2}=t_{1}
$$

and then (3.41) is satisfied.

Using (3.36) and (3.41) from (3.19) we can easily obtain that

$$
\int_{\mathbb{R}}\left(\frac{\partial \theta}{\partial x}\right)^{2}(x, t) d x<2 K_{3}\left(M_{1}\right) \text { for } 0<t \leq t_{1} .
$$

Now, we introduce the function $\Psi$ by

$$
\Psi(\theta(x, t))=\int_{1}^{\theta(x, t)} \sqrt{s-1-\ln s} d s .
$$

From (2.17) follows that $\theta(x, t) \rightarrow 1$ as $|x| \rightarrow \infty$ and hence

$$
\Psi(\theta(x, t)) \rightarrow 0 \text { as }|x| \rightarrow \infty .
$$

Consequently,

$$
\begin{aligned}
\psi(\theta(x, t)) & \leq|\psi(\theta(x, t))|=\left|\int_{1}^{\theta(x, t)} \frac{d}{d s} \psi(s) d s\right| \\
& =\left|\int_{-\infty}^{x} \sqrt{\theta(x, t)-1-\ln \theta(x, t)} \frac{\partial \theta(x, t)}{\partial x} d x\right| \\
& \leq\left(\int_{\mathbb{R}}(\theta(x, t)-1-\ln \theta(x, t)) d x\right)^{\frac{1}{2}}\left(\int_{\mathbb{R}}\left(\frac{\partial \theta}{\partial x}\right)^{2}(x, t) d x\right)^{\frac{1}{2}} .
\end{aligned}
$$

Taking into account (3.36), (3.50) and (3.1) from (3.53) we get

$$
\max _{0 \leq \theta(x, t) \leq M_{1}} \psi(\theta(x, t))=\psi\left(\bar{\theta}\left(t_{1}\right)\right)=\psi\left(M_{1}\right) \leq\left(2 K_{3}\left(M_{1}\right) E_{1}\right)^{\frac{1}{2}}
$$


Or

$$
\int_{1}^{M_{1}} \sqrt{s-1-\ln s} d s-\left(2 K_{3}\left(M_{1}\right) E_{1}\right)^{\frac{1}{2}} \leq 0 .
$$

Since this inequality contradicts $(2.26)$, it remains to assume that $t_{1}=T$. Hence we have

Lemma 3.4. For each $T>0$ we have

$$
\begin{gathered}
\theta(x, t) \leq M_{1}, \quad(x, t) \in \Pi, \\
\int_{\mathbb{R}}\left(\frac{\partial v}{\partial x}\right)^{2}(x, t) d x \leq K_{4}\left(M_{1}\right), \quad 0 \leq t \leq T, \\
\int_{\mathbb{R}}\left(\frac{\partial \theta}{\partial x}\right)^{2}(x, t) d x \leq 2 K_{3}\left(M_{1}\right), \quad 0 \leq t \leq T .
\end{gathered}
$$

Proof. These conclusions follow from (3.36), (3.41) and (3.50) directly.

LEMma 3.5. The following inequalities hold true:

$$
\begin{gathered}
0<\underline{u} \leq \frac{1}{\rho(x, t)} \leq \bar{u}, \quad(x, t) \in \Pi, \\
\sup _{(x, t) \in \Pi}|v(x, t)| \leq \sqrt{8 E_{1} K_{4}\left(M_{1}\right)}, \\
\theta(x, t) \geq h>0, \quad(x, t) \in \Pi
\end{gathered}
$$

where $\underline{u}$ and $\bar{u}$ are defined by (2.22)-(2.24) and a constant $h$ depends only on the data of problem (2.1)-(2.8).

Proof. Because the quantity $\underline{u}_{1}(\bar{\theta}(t))$ in Lemma 3.2 decreases with increasing $\bar{\theta}(t)$, while $\bar{u}_{1}(\bar{\theta}(t))$ increases, it follows from (3.2) and (3.56) that (3.59) is satisfied.

Using the inequality

$$
v^{2}=2 \int_{-\infty}^{x} v \frac{\partial v}{\partial x} d x \leq 2\left(\int_{\mathbb{R}} v^{2} d x\right)^{\frac{1}{2}}\left(\int_{\mathbb{R}}\left(\frac{\partial v}{\partial x}\right)^{2} d x\right)^{\frac{1}{2}}
$$

and estimations (3.1) and (3.57) we get immediately (3.60). From (3.53), (3.56), (3.58) and (3.1) we have for $\theta(x, t) \leq 1$ that the following holds

$$
\int_{\theta(x, t)}^{1} \sqrt{s-1-\ln s} d s \leq\left(2 K_{3}\left(M_{1}\right) E_{1}\right)^{\frac{1}{2}}<\int_{0}^{1} \sqrt{s-1-\ln s} d s
$$

because of (2.26). Hence we conclude that there exists the constant $h>0$ such that $\theta(x, t) \geq h$. 
LEMMA 3.6. For each $T>0$ we have

$$
\begin{gathered}
\int_{0}^{T} \int_{\mathbb{R}}\left(\frac{\partial v}{\partial x}\right)^{2} d x d \tau \leq K_{5}, \\
\int_{0}^{T} \int_{\mathbb{R}}\left(\frac{\partial \theta}{\partial x}\right)^{2} d x d \tau \leq K_{6}, \\
\int_{0}^{T} \int_{\mathbb{R}}\left(\frac{\partial \rho}{\partial x}\right)^{2} d x d \tau \leq K_{7}, \\
\int_{\mathbb{R}}\left(\frac{\partial \rho}{\partial x}\right)^{2} d x \leq K_{8}, \quad t \in[0, T] \\
\int_{0}^{T} \int_{\mathbb{R}}\left(\frac{\partial^{2} \theta}{\partial x^{2}}\right)^{2} d x d \tau \leq K_{9}, \\
\int_{0}^{T} \int_{\mathbb{R}}\left(\frac{\partial^{2} v}{\partial x^{2}}\right)^{2} d x d \tau \leq K_{10},
\end{gathered}
$$

where the constants $K_{5}, K_{6}, K_{7}, K_{8}, K_{9}, K_{10} \in \mathbb{R}^{+}$are independent of $T$.

Proof. Taking into account (3.59) and (3.61) from (3.1), (3.11), (3.19) and (3.31) we get easily (3.64)-(3.69).

\section{Proof of Theorem 2.1}

In the following we use the results of Section 3. It is important to remark that all the estimates obtained above are preserved in the domain $\Pi=\mathbb{R} \times] 0, T[$ for each $T>0$.

The conclusions of Theorem 2.1 are immediate consequences of the following lemmas.

LEMma 4.1. The following relations hold true:

$$
\int_{\mathbb{R}}\left(\frac{\partial v}{\partial x}\right)^{2}(x, t) d x \rightarrow 0, \quad \int_{\mathbb{R}}\left(\frac{\partial \theta}{\partial x}\right)^{2}(x, t) d x \rightarrow 0
$$

when $t \rightarrow \infty$.

Proof. Let $\varepsilon>0$ be arbitrary. With the help of (3.64), (3.65) and (3.66) we conclude that there exists $t_{0}>0$ such that

$$
\int_{t_{0}}^{t} \int_{\mathbb{R}}\left(\frac{\partial v}{\partial x}\right)^{2} d x d \tau<\varepsilon, \int_{t_{0}}^{t} \int_{\mathbb{R}}\left(\frac{\partial \theta}{\partial x}\right)^{2} d x d \tau<\varepsilon, \int_{t_{0}}^{t} \int_{\mathbb{R}}\left(\frac{\partial \rho}{\partial x}\right)^{2} d x d \tau<\varepsilon
$$


for $t>t_{0}$, and

$$
\int_{\mathbb{R}}\left(\frac{\partial v}{\partial x}\right)^{2}\left(x, t_{0}\right) d x<\varepsilon, \quad \int_{\mathbb{R}}\left(\frac{\partial \theta}{\partial x}\right)^{2}\left(x, t_{0}\right) d x<\varepsilon
$$

Similarly to (3.19), we have

$$
\begin{aligned}
& \frac{1}{2} \int_{\mathbb{R}}\left[\left(\frac{\partial v}{\partial x}\right)^{2}+\left(\frac{\partial \theta}{\partial x}\right)^{2}\right] d x \\
&+\frac{8 \bar{u}}{(D \underline{u})^{2}} \int_{t_{0}}^{t}\left(\int_{\mathbb{R}}\left(\frac{\partial v}{\partial x}\right)^{2} d x\right)^{2}\left[K_{4}(\bar{\theta}(\tau))-\int_{\mathbb{R}}\left(\frac{\partial v}{\partial x}\right)^{2} d x\right] d \tau \\
& \quad+\frac{D}{8} \int_{t_{0}}^{t} \int_{\mathbb{R}} \rho\left(\frac{\partial^{2} \theta}{\partial x^{2}}\right)^{2} d x d \tau \\
& \leq \frac{1}{2} \int_{\mathbb{R}}\left[\left(\frac{\partial v}{\partial x}\right)^{2}+\left(\frac{\partial \theta}{\partial x}\right)^{2}\right]\left(x, t_{0}\right) d x+K^{2} \int_{t_{0}}^{t} \int_{\mathbb{R}} \rho\left(\frac{\partial \theta}{\partial x}\right)^{2} d x d \tau \\
&+\left(16 K_{1}(\bar{\theta}(t))\right)^{2} \frac{\bar{u}}{\underline{u^{2}}} \int_{t_{0}}^{t} \int_{\mathbb{R}}\left(\frac{\partial v}{\partial x}\right)^{2} d x d \tau \\
&+K^{2} \int_{t_{0}}^{t} \int_{\mathbb{R}} \frac{\theta^{2}}{\rho}\left(\frac{\partial \rho}{\partial x}\right)^{2} d x d \tau+\frac{K^{2}}{D} \int_{t_{0}}^{t} \int_{\mathbb{R}} \theta^{2} \rho\left(\frac{\partial v}{\partial x}\right)^{2} d x d \tau \\
&+\frac{128 D K_{1}^{2}(\bar{\theta}(t)) \bar{u}}{\underline{u}^{2}} \int_{t_{0}}^{t} \int_{\mathbb{R}}\left(\frac{\partial \theta}{\partial x}\right)^{2} d x d \tau
\end{aligned}
$$

Taking into account (3.56), (3.59) and (4.2)-(4.3) from (4.4) we obtain

$$
\int_{\mathbb{R}}\left[\left(\frac{\partial v}{\partial x}\right)^{2}+\left(\frac{\partial \theta}{\partial x}\right)^{2}\right] d x \leq K_{11} \varepsilon \text { for } t>t_{0}
$$

where $K_{11}$ depends only on the data of our problem and does not depend on $t_{0}$. Hence relations (4.1) hold.

LEMMA 4.2. We have

$$
v(x, t) \rightarrow 0, \quad \theta(x, t) \rightarrow 1
$$

when $t \rightarrow \infty$, uniformly with respect to all $x \in \mathbb{R}$.

Proof. We have (see (3.62) and (3.53))

$$
v^{2}(x, t) \leq 2\left(\int_{\mathbb{R}} v^{2}(x, t) d x\right)^{\frac{1}{2}}\left(\int_{\mathbb{R}}\left(\frac{\partial v}{\partial x}\right)^{2}(x, t) d x\right)^{\frac{1}{2}}
$$

(4.8) $|\psi(\theta(x, t))| \leq\left(\int_{\mathbb{R}}(\theta(x, t)-1-\ln \theta(x, t)) d x\right)^{\frac{1}{2}}\left(\int_{\mathbb{R}}\left(\frac{\partial \theta}{\partial x}\right)^{2} d x\right)^{\frac{1}{2}}$. 
Taking into account (3.1) from (4.7) and (4.8) we get

$$
\begin{gathered}
v^{2}(x, t) \leq 2\left(2 E_{1}\right)^{\frac{1}{2}}\left(\int_{\mathbb{R}}\left(\frac{\partial v}{\partial x}\right)^{2}(x, t) d x\right)^{\frac{1}{2}}, \\
|\psi(\theta(x, t))| \leq E_{1}^{\frac{1}{2}}\left(\int_{\mathbb{R}}\left(\frac{\partial \theta}{\partial x}\right)^{2} d x\right)^{\frac{1}{2}} .
\end{gathered}
$$

Using (4.1) and property (3.52) of the function $\psi$ we can easily obtain that (4.6) holds.

LEMma 4.3. We have

$$
\int_{\mathbb{R}}\left(\frac{\partial \rho}{\partial x}\right)^{2}(x, t) d x \rightarrow 0
$$

when $t \rightarrow \infty$.

Proof. From (3.66) and (3.69) we conclude that for $\varepsilon>0$ exists $t_{0}>0$ such that

$$
\int_{t_{0}}^{t} \int_{\mathbb{R}}\left(\frac{\partial \rho}{\partial x}\right)^{2} d x d \tau<\varepsilon, \int_{t_{0}}^{t} \int_{\mathbb{R}}\left(\frac{\partial^{2} v}{\partial x^{2}}\right)^{2} d x d \tau<\varepsilon, \int_{\mathbb{R}}\left(\frac{\partial \rho}{\partial x}\right)^{2}\left(x, t_{0}\right) d x<\varepsilon
$$

for $t>t_{0}$. Now, from (2.1) we get the equality

$$
\frac{\partial}{\partial t}\left(\frac{\partial}{\partial x}\left(\frac{1}{\rho}\right)\right)=\frac{\partial^{2} v}{\partial x^{2}}
$$

Multiplying (4.13) by $\frac{\partial}{\partial x}\left(\frac{1}{\rho}\right)$ and integrating over $\mathbb{R}$ and $] t_{0}, t[$ we obtain (4.14)

$$
\frac{1}{2} \int_{\mathbb{R}} \frac{1}{\rho^{4}}\left(\frac{\partial \rho}{\partial x}\right)^{2} d x=-\int_{t_{0}}^{t} \int_{\mathbb{R}} \frac{1}{\rho^{2}} \frac{\partial \rho}{\partial x} \frac{\partial^{2} v}{\partial x^{2}} d x d \tau+\frac{1}{2} \int_{\mathbb{R}} \frac{1}{\rho^{4}}\left(\frac{\partial \rho}{\partial x}\right)^{2}\left(x, t_{0}\right) d x .
$$

Using the Young's inequality and (3.59) from (4.14) we find out

$$
\begin{aligned}
\frac{\underline{u}^{4}}{2} \int_{\mathbb{R}}\left(\frac{\partial \rho}{\partial x}\right)^{2} d x \leq & \bar{u}^{2} \int_{t_{0}}^{t} \int_{\mathbb{R}}\left(\frac{\partial \rho}{\partial x}\right)^{2} d x d \tau+\bar{u}^{2} \int_{t_{0}}^{t} \int_{\mathbb{R}}\left(\frac{\partial^{2} v}{\partial x^{2}}\right)^{2} d x d \tau \\
& +\frac{\underline{u}^{4}}{2} \int_{\mathbb{R}}\left(\frac{\partial \rho}{\partial x}\right)^{2}\left(x, t_{0}\right) d x
\end{aligned}
$$

With the help of (4.12) from (4.15) we get (4.11).

LEMMA 4.4. We have

$$
\rho(x, t) \rightarrow 1
$$

when $t \rightarrow \infty$, uniformly with respect to $x \in \mathbb{R}$. 
Proof. Similarly as for the function $\psi(\theta)$, we have

$$
\begin{aligned}
\psi\left(\frac{1}{\rho}\right) & =\int_{1}^{\frac{1}{\rho}} \sqrt{s-1-\ln s} d s \\
& \leq\left(\int_{\mathbb{R}}\left(\frac{1}{\rho}-1-\ln \frac{1}{\rho}\right) d x\right)^{\frac{1}{2}}\left(\int_{\mathbb{R}} \frac{1}{\rho^{2}}\left(\frac{\partial \rho}{\partial x}\right)^{2} d x\right)^{\frac{1}{2}} .
\end{aligned}
$$

Taking into account (3.1) from (4.17) follows

$$
\psi\left(\frac{1}{\rho}\right) \leq\left(K E_{1}\right)^{\frac{1}{2}} \bar{u}\left(\int_{\mathbb{R}}\left(\frac{\partial \rho}{\partial x}\right)^{2} d x\right)^{\frac{1}{2}}
$$

and with the help of (4.11) we conclude (4.16).

\section{REFERENCES}

[1] S. N. Antontsev, A. V. Kazhykhov and V. N. Monakhov, Boundary value problems in mechanics of nonhomogeneous fluids, North-Holland, Amsterdam, 1990.

[2] R. Dautray and J. L. Lions, Mathematical analysis and numerical methods for science and techonology. Vol. 2, Springer-Verlag, Berlin, 1988.

[3] R. Dautray and J. L. Lions, Mathematical analysis and numerical methods for science and techonology. Vol. 5, Springer-Verlag, Berlin, 1992.

[4] Ya. I. Kanel', Cauchy problem for equations of gas dynamics with viscosity, Sibirsk. Mat. Zh. 20 (1979), 293-306.

[5] N. Mujaković, One-dimensional flow of a compressible viscous micropolar fluid: The Cauchy problem, Math. Commun. 10 (2005), 1-14.

[6] N. Mujaković, Uniqueness of a solution of the Cauchy problem for one-dimensional compressible viscous micropolar fluid model, Appl. Math. E-Notes 6 (2006), 113-118.

N. Mujaković

Department of Mathematics

University of Rijeka

Omladinska 14, 51000 Rijeka

Croatia

E-mail: mujakovic@inet.hr

I. Dražić

Faculty of Engineering

University of Rijeka

Vukovarska 58, 51000 Rijeka

Croatia

E-mail: idrazic@riteh.hr

Received: 2.10.2009.

Revised: 26.2.2010. 